\title{
Prevalência de Afastamentos por Transtornos Mentais e do Comportamento Relacionados ao Trabalho em Professores
}

Prevalencia de Bajas por Trastornos Mentales Comunes y de la Conducta Relacionados al Trabajo en Profesores

Prevalence of Sick Leave at Work by Mental and Behavioral Disorders of Teachers

Mary Sandra Carlotto

ORCID: http://orcid.org/0000-0003-2336-5224

Universidade do Vale do Rio dos Sinos, Rio Grande do Sul/ Brasil

Sheila Gonçalves Câmara

ORCID: http://orcid.org/0001-6761-7644

Universidade Federal de Ciências da Saúde de Porto Alegre, Rio Grande do Sul/ Brasil

Jaqueline Vidal Batista

ORCID: http://orcid.org/0002-8107-9763

Universidade Federal da Paraíba, Paraíba/ Brasil

Gabriela Azeredo Schneider

ORCID: https://orcid.org/0000-0002-6541-7937

Universidade do Vale do Rio dos Sinos, Rio Grande do Sul/ Brasil

\begin{abstract}
Declaração de Direito Autoral
A submissão de originais para este periódico implica na transferência, pelos autores, dos direitos de publicação impressa e digital. Os direitos autorais para os artigos publicados são do autor, com direitos do periódico sobre a primeira publicação. Os autores somente poderão utilizar os mesmos resultados em outras publicações indicando claramente este periódico como o meio da publicação original. Em virtude de sermos um periódico de acesso aberto, permite-se o uso gratuito dos artigos em aplicações educacionais e científicas desde que citada a fonte conforme a licença CC-BY da Creative Commons.
\end{abstract}

\section{Resumo}

O objetivo do estudo foi identificar a prevalência dos afastamentos do trabalho por Transtornos Mentais e Comportamentais Relacionados Ao Trabalho (TMCRT) em professores. Foi utilizado um delineamento transversal das licenças médicas dos professores entre os anos de 2012 a 2016 . A população em estudo foram todos os professores de escolas municipais de um município da Região Metropolitana de Porto Alegre/RS (n=2.181). A amostra foi constituída por 246 licenças de afastamento por motivo de saúde relacionado ao trabalho dentre 116 professores, no período em estudo. A prevalência de TMRC foi de $11 \%$ em relação ao número de trabalhadores da educação do município e $8 \%$ em relação aos motivos de afastamento em geral. Os resultados evidenciaram maior frequência de transtornos neuróticos, transtornos relacionados com o "stress" e transtornos somatoformes $(52,4 \%)$, seguido de transtornos do humor $(42,7 \%)$. Esses dados indicam que os transtornos mentais em professores constituem uma realidade preocupante que necessita monitoramento constante para o estabelecimento de políticas públicas de promoção da saúde e prevenção de agravos mentais à saúde dos professores.

Palavras-chaves: Transtornos mentais; Absenteísmo; Segurança e saúde ocupacional; Professores. 


\title{
Resumen
}

El objetivo del estudio fue identificar la prevalencia de bajas en el trabajo por Trastornos Mentales y Conductuales Relacionados al Trabajo (TMCRT) en profesores. Fue utilizado un diseño trasversal de las bajas medicas de los profesores entre los años 2012 y 2016. La población del estudio fueron todos los profesores de escuelas municipales de un municipio de la región metropolitana de Porto Alegre/RS $(n=2.181)$. La muestra fue constituida por 246 bajas por motivos de salud relacionados al trabajo presentadas por 116 profesores en el periodo en estudio. La prevalencia de TMCRT fue de $11 \%$ en términos del número de trabajadores de la educación en la municipalidad, y $8 \%$ en términos de las bajas generales. Los resultados evidenciaron mayor frecuencia de trastornos neuróticos, trastornos relacionados al "stress" y trastornos somatoformes (52,4\%), seguidos de trastornos del humor $(42,7 \%)$. Los datos indican que los trastornos mentales en profesores representan una realidad preocupante que requiere monitoreo constante para el establecimiento de políticas públicas de promoción de la salud y prevención de enfermedades mentales a la salud de los profesores.

Palabras claves: Trastornos mentales; Absentismo; Seguridad y salud ocupacional; Profesores.

\begin{abstract}
The objective of the present study was to identify the prevalence of work-related disorders due to Mental Disorders and Work-Related Behavior in teachers (TMCRT). It was used a cross-sectional design of the medical licenses of teachers between the years 2012 and 2016. The study population was all teachers from municipal schools in a city in the metropolitan region of Porto Alegre/RS $(n=2.181)$. The sample consisted of 246 absences from 116 teachers in the studied period. The prevalence of TMRC was $11 \%$ in terms of the number of education workers in the municipality and $8 \%$ in relation to absence reasons in general. Results showed a higher frequency of neurotic disorders, stress related disorders and somatoform disorders $(52.4 \%)$ followed by mood disorders $(42.7 \%)$. Data indicates that the mental disorders in teachers constitute a worrisome reality that needs constant monitoring for the establishment of public policies of health promotion and prevention of teachers' mental health disorders.
\end{abstract}

Keywords: Mental disorders; Absenteeism; Occupational safety and health, Teachers.

\section{Introdução}

O absenteísmo laboral consiste em um grave problema para as organizações de trabalho, gestores e trabalhadores e sua ocorrência implica em interrupção dos processos de trabalho, necessidade de redistribuição de tarefas, gerando sobrecarga de trabalho entre os colegas, perda de produtividade (Aguiar \& Oliveira, 2009; Böckerman \& Laukkanen, 2010; Ybema, Smulders, \& Bongers, 2010) e qualidade dos serviços prestados (Affonso, 2014; Munro, 2007; Santi, Barbieri, \& Cheade, 2018). As faltas ao trabalho, na maioria das vezes, podem indicar a existência de problemas relacionados à saúde do trabalhador (Aguiar \& Oliveira, 2009).
Embora as condições gerais de saúde da população mundial tenham apresentado melhora nos últimos 100 anos, as taxas de afastamento do trabalho por doença têm aumentado nos países industrializados, desde a década de 50, o que repercute em altos custos às economias em todo o mundo (SeligmannSilva, 2009). Os problemas de saúde relacionados com o trabalho resultam em perda econômica que varia de 4 a $6 \%$ do Produto Interno Bruto para a maioria dos países (Organização Mundial de Saúde [OMS], 2014).

As diversas mudanças ocorridas no mundo do trabalho nas últimas décadas têm afetado a saúde dos trabalhadores de diferentes 
maneiras, podendo ser destacadas as condições precárias trabalho, instabilidade no emprego, poucas possibilidades de desenvolvimento e crescimento profissional. Além disso, as formas agressivas de organização do trabalho estabelecem metas difíceis de atingir, gerando desconforto entre os trabalhadores. Todos estes fatores podem contribuir para o adoecimento do trabalhador (Alencar \& Valença, 2016).

As atividades de trabalho, quando patogênicas, podem atingir o corpo dos trabalhadores ocasionando disfunções e lesões biológicas e reações psíquicas (Ministério da Saúde do Brasil, 2001) com destaque para o aumento de adoecimentos relacionados ao trabalho expressos por meio de transtornos mentais (McDaid, 2008; Wynne et al., 2014).

Estudos atuais revelam aumento no número de pessoas que adoecem e se afastam do trabalho pelos mais variados motivos de saúde, porém os Transtornos Mentais e do Comportamento têm sido os mais prevalentes (Falavigna \& Carlotto, 2013; Seligmann-Silva, 2009) e se constituem em uma das maiores causas de afastamento de longo prazo do trabalho, aposentadorias antecipadas e por invalidez (Stansfeld, North, White, \& Marmot, 1995; Wynne et al., 2014).

$\mathrm{Na}$ Alemanha verificou-se um aumento de, 2,5 vezes de aposentadorias antecipadas devido a problemas de saúde mental entre os anos de 1989 a 2012, tendo sido observada a mesma tendência de aumento nos demais países da comunidade europeia. No Reino Unido foi verificado um aumento de afastamentos devido a problemas de saúde mental relacionados ao estresse em proporção maior do que os afastamentos por problemas físicos (Wynne et al., 2014).

\section{Os Transtornos Mentais e} Comportamentais são caracterizados como condições clinicamente significativas, nas quais estão presentes alterações do modo de pensar e do humor (emoções) e comportamentos associados com angústia pessoal e/ou deterioração do funcionamento (Ministério da Saúde do Brasil, 2001). Os
Transtornos Mentais e Comportamentais Relacionados Ao Trabalho (TMCRT) correspondem a diversos diagnósticos presentes na Classificação Internacional de Doenças (CID-10). Desde 1999, são oficialmente reconhecidos pelo Ministério da Saúde e pelo Ministério da Previdência e Assistência Social (Seligmann-Silva, Bernardo, Maeno, \& Kato, 2010). De acordo com os autores, diversos fatores presentes no atual contexto de trabalho podem impactar a saúde mental dos trabalhadores, por exemplo, a exposição a agentes tóxicos, a altos níveis de ruído, a situações de risco à integridade física, a formas de organização do trabalho e politicas de gestão que não atentam aos limites físicos e psíquicos do trabalhador.

Estudo realizado por Costa (2017) cujo objetivo foi identificar o perfil epidemiológico dos casos de TMCRT notificados pelo Sistema de Informação de Agravos de Notificação (SINAN), na região Nordeste do Brasil, no período de 2007 a 2016, identificou que dos nove Estados da Região Nordeste, o Rio Grande do Norte foi o que apresentou o maior número de casos notificados $(36,5 \%)$, seguido por Pernambuco $(24,8 \%)$ e Bahia $(19,2 \%)$. Dos 1.591 casos de TMRT analisados entre 2007 e 2016, os transtornos neuróticos, relacionados ao stress e os somatoformes (F40-F48) e os transtornos do humor (F30-F39) apresentaram a maior frequência de notificações, com $69,2 \%$ e $24 \%$ respectivamente.

Investigação sobre a variação do perfil dos benefícios previdenciários por transtornos mentais e comportamentais e sua relação com o trabalho com dados secundários da Previdência Social brasileira de 2008 a 2011, realizado por Silva Junior \& Fischer (2014), identificou que os transtornos mentais permaneceram como a terceira causa das concessões de benefícios. Os resultados identificaram também um aumento médio anual de $0,3 \%$ de novas concessões, com queda de $2,5 \%$ da incidência média anual. Foram considerados relacionados ao trabalho $6,2 \%$ dos casos, na média, principalmente decorrentes de transtornos de humor. 
$\mathrm{O}$ adoecimento provoca a diminuição da força produtiva economicamente ativa, gerando importantes custos ao Estado, assim como a exclusão social, agravando ainda mais o sofrimento crônico dos indivíduos adoecidos e promovendo, assim, um ciclo de desgaste (Silva Junior \& Fischer, 2014). De acordo com Ybema, Smulders e Bonger (2010), frequentes faltas ao trabalho podem significar que o trabalhador precisa de tempo para se recuperar das tensões no trabalho e sua ausência prolongada pode ser um indicador de problemas mais graves.

A categoria profissional docente é considerada como uma das ocupações com alto risco de desgaste e adoecimento e tem sido alvo de diversos estudos avaliando a prevalência e fatores associados aos transtornos mentais (Araújo \& Carvalho, 2009; Carlotto \& Câmara, 2015; Gasparini, Barreto, \& Assunção, 2006; Khan et al., 2017; Soldatelli, 2011) e sua relação com o afastamento do trabalho em professores (Batista, Carlotto, Coutinho, \& Augusto, 2010; Maia, 2014).

$\mathrm{O}$ perfil epidemiológico dos Transtornos Mentais e do Comportamento (TMC) de servidores públicos do estado de Santa Catarina, afastados por Licenças para Tratamento de Saúde (LTS) entre 2010 e 2013, identificado por Baasch, Trevisan e Cruz (2017), revelou que 40,14\% dos afastamentos foram decorrentes de TMC. A maior prevalência em trabalhadores foi encontrada nos setores de educação (39\%). Entre servidores públicos, a categoria dos professores foi a que mais se afastou por problemas de saúde e a Secretaria de Estado da Educação foi a responsável pelo maior número de LTS em decorrência de TMC.

A alta prevalência dos problemas de saúde encontrados em estudo realizado com professores por Araújo e Carvalho (2009) associou-se à elevada demanda psicológica exigida na execução das atividades, ao baixo controle sobre o próprio trabalho, ao maior tempo de trabalho como professor, à elevada carga horária semanal, aos múltiplos empregos e a uma série de características relativas ao ambiente e organização do trabalho docente, tais como ritmo de trabalho, ambiente em condições inadequadas, relações interpessoais estressantes, entre outros aspectos. Investigação realizada por Morais, Souza e Santos (2018) com professores de escolas públicas revela um contexto de trabalho precarizado devido às condições inadequadas do ambiente físico de trabalho, à falta de material para desenvolver as atividades docentes e um trabalho intensificado com horários que extrapolam a carga horária contratual que se estendem ao tempo reservado ao descanso e lazer.

Outros estressores têm sido associados a problemas de saúde, como a falta de interesse e a indisciplina dos alunos (Carlotto \& Palazzo, 2006; Sales \& Freitas, 2018), a falta de estrutura, a falta de diálogo e o autoritarismo, tanto da coordenação quanto da direção escolar, o individualismo por parte de colegas, os baixos salários (Costa \& Rocha, 2013), as turmas de difícil manejo, a hostilidade dos pais, a pouca assistência, o pouco apoio de administradores e gestores públicos (Khan, 2014; Sprenger, 2011) e a inexpressiva participação nas políticas e no planejamento institucional (Melo, Rego, Saldanha, Flor, \& Maracaja, 2015). Os afastamentos de professores por problemas de saúde podem estar relacionados diretamente com as condições de trabalho (Santos \& Wanzinack, 2017).

A saúde docente ainda é tratada como uma questão secundária nas preocupações do setor da educação, tanto por parte de gestores como dos próprios professores (Araújo \& Carvalho, 2009). Os autores complementam afirmando que a falta de reconhecimento do adoecimento e do estabelecimento de sua relação com o trabalho tem como maior consequência a manutenção de situações prejudiciais à saúde, contribuindo para o aumento do adoecimento desta categoria profissional assim como para o abandono da profissão.

O absenteísmo decorrente de doenças é um problema de saúde pública e apresenta um 
impacto em termos sociais, organizacionais e individuais acarretando importantes gastos sociais e deve ser administrado para viabilizar políticas adequadas de prevenção (Schreuder, Roelen, Koopmans, Moen \& Groothoff, 2010). Seu monitoramento e a avaliação sistemática podem subsidiar a tomada de decisão gerencial, assim como o aperfeiçoamento de políticas de recursos humanos, programas de prevenção à saúde do trabalhador e a melhoria da qualidade de vida no trabalho (Estorce \& Kurcgant, 2011).

Assim, identificar o perfil de adoecimento é fundamental para o planejamento de politicas públicas de promoção de saúde e prevenção de agravos (Borrell \& Artazcoz, 2008; Leão, BarbosaBranco, Rassi Neto, Ribeiro, \& Turchi, 2015). Neste sentido, o presente estudo objetivou identificar a prevalência dos afastamentos do trabalho por transtornos mentais e do comportamento em professores no período de 2012 a 2016, descrevendo os transtornos mentais mais frequentes e sua distribuição nesse período de tempo.

\section{Método \\ Delineamento}

Estudo descritivo retrospectivo, que utilizou dados secundários.

\section{População/amostra}

A população em estudo foi composta por 2.181 professores de escolas públicas municipais de uma cidade da região metropolitana de Porto Alegre, Estado do Rio Grande do Sul, entre os anos de 2012 e 2016.

A cidade possui uma população de 229.678 habitantes e uma taxa de urbanização de $99,7 \%$ de acordo com dados do IBGE no ano 2016. A área urbana é dividida em 24 bairros. Possui 45 escolas municipais, sendo 10 de Educação Infantil e 35 de Ensino Fundamental. O censo escolar Censo Escolar/INEP-2016 contabilizou 18.555 alunos.

A amostra foi constituída por 116 professores que apresentaram 246 licenças por TMCRT, de um total de 2.931 licenças nos anos estudados, conforme registrado no Setor de Recursos Humanos. O período do levantamento deveu-se ao fato de que os registros passaram a ser informatizados a partir de 2012.

\section{Procedimentos de coleta de dados}

Primeiramente, foi realizado um contato com a Secretária de Municipal de Educação, momento no qual foram expostos os objetivos do estudo e foi obtida autorização para a sua realização. Foi esclarecido tratar-se de uma pesquisa sem quaisquer efeitos avaliativos individuais e/ou institucionais e que seus dados seriam anônimos e confidenciais. O estudo atendeu as recomendações éticas e possui aprovação do Comitê de Ética e Pesquisa da universidade (n.02641012.5.0000.5336).

Os dados foram disponibilizados pelo responsável do Setor de Recursos Humanos sem a coluna de identificação funcional do trabalhador. Após análise preliminar, contatouse que seus campos apresentaram adequada completude e fidedignidade para a realização do levantamento dos dados. Foram selecionadas as seguintes variáveis relativas aos professores afastados: sexo, idade, tempo de trabalho, dias de afastamento e diagnóstico médico (CID-10). sexo, idade, tempo de trabalho, dias de afastamento e diagnóstico médico (CID-10).

\section{Procedimentos de análise de dados}

Para atender ao objetivo do estudo, foi realizada estatística descritiva para os afastamentos por Transtornos Mentais e do Comportamento, por meio da distribuição de frequências. Foram, então, calculadas as taxas de afastamento para os anos de 2012 a 2016, segundo Capítulo V - Transtornos mentais e comportamentais (F00-F99) da Classificação Internacional de Doença (CID-10), para cada um dos 11 grupos, conforme descritos a seguir (Ministério da Saúde do Brasil, 2001).

Grupo 1 - Transtornos mentais 
orgânicos, inclusive os sintomáticos (F00F09): Este agrupamento se constitui de uma série de transtornos mentais que possuem em comum uma etiologia demonstrável tal como doença ou lesão cerebral ou outro comprometimento que leva à disfunção cerebral. Tal disfunção pode ser primária, como em doenças, lesões e comprometimentos que afetam o cérebro de maneira direta e seletiva ou secundária como patologias e transtornos sistêmicos que envolvem dano ao cérebro apenas como um dos múltiplos órgãos ou sistemas orgânicos envolvidos.

Grupos 2 - Transtornos mentais e comportamentais devidos ao uso de substância psicoativa (F10-F19): Grupo constituído por transtornos que diferem entre si pela gravidade variável e por sintomatologia diversa, mas que têm em comum o fato de serem todos atribuídos ao uso de uma ou de várias substâncias psicoativas, prescritas ou não por um médico.

Grupo 3 - Esquizofrenia, transtornos esquizotípicos e transtornos delirantes (F20F29): Este agrupamento tem a esquizofrenia como categoria mais importante deste grupo de transtornos, o transtorno esquizotípico e os transtornos delirantes persistentes e um grupo maior de transtornos psicóticos agudos e transitórios.

Grupo 4 - Transtornos do humor/afetivos (F30-F39): Neste agrupamento, a perturbação fundamental é uma alteração do humor ou do afeto, no sentido de uma depressão (com ou sem ansiedade associada) ou de uma elação. A alteração do humor em geral vem associada a uma modificação do nível global de atividade, e a maioria dos outros sintomas são secundários a estas alterações do humor e da atividade e facilmente compreensíveis no contexto destas alterações. A maioria destes transtornos tende a ser recorrentes e a ocorrência dos episódios individuais pode frequentemente estar relacionada a situações ou eventos estressantes.

Grupo 5 - Transtornos neuróticos, transtornos relacionados com o "stress" e transtornos somatoformes (F40-F48): Grupo de transtornos em que ocorre ansiedade desencadeada exclusiva ou essencialmente por situações nitidamente determinadas que não apresentam atualmente nenhum perigo real.

Grupo 6 -Síndromes comportamentais associadas a disfunções fisiológicas e a fatores físicos (F50-F59): grupo constituído por transtornos da alimentação, transtornos nãoorgânicos do sono devidos a fatores emocionais, disfunção sexual, não causada por transtorno ou doença orgânica, transtornos mentais e comportamentais associados ao puerpério, não classificados em outra parte, fatores psicológicos ou comportamentais associados a doença ou a transtornos classificados em outra parte, abuso de substâncias que não produzem dependência e síndromes comportamentais associados a transtornos das funções fisiológicas e a fatores físicos, não especificadas.

Grupo 7 -Transtornos da personalidade e do comportamento do adulto (F60-F69): Este agrupamento compreende diversos estados e tipos de comportamento clinicamente significativos que tendem a persistir e são a expressão característica da maneira de viver do indivíduo e de seu modo de estabelecer relações consigo próprio e com os outros. Alguns destes estados e tipos de comportamento aparecem precocemente durante o desenvolvimento individual sob a influência conjunta de fatores constitucionais e sociais, enquanto outros são adquiridos mais tardiamente durante a vida. Os transtornos específicos da personalidade, os transtornos mistos e outros transtornos da personalidade, e as modificações duradouras da personalidade representam modalidades de comportamento enraizadas e duradouras, que se manifestam sob a forma de reações inflexíveis a situações pessoais e sociais de natureza muito variada. Eles representam desvios extremos ou significativos das percepções, dos pensamentos, das sensações e particularmente das relações com os outros em relação àquelas de um indivíduo médio de uma dada cultura. Tais tipos de comportamento são geralmente estáveis e englobam múltiplos domínios do 
comportamento e do funcionamento psicológico. Frequentemente estão associados a sofrimento subjetivo e a comprometimento de intensidade variável do desempenho social.

Grupo 8 - Retardo mental (F70-F79): Grupo caracterizado pela ocorrência de parada ou pelo desenvolvimento incompleto do funcionamento intelectual, marcados essencialmente por um comprometimento, durante o período de desenvolvimento, das faculdades que determinam o nível global de inteligência, isto é, das funções cognitivas, de linguagem, da motricidade e do comportamento social. O retardo mental pode acompanhar um outro transtorno mental ou físico, ou ocorrer de modo independentemente.

Grupo 9 - Transtornos do desenvolvimento psicológico (F80-F89): Os transtornos classificados neste agrupamento possuem em comum o fato do início situado obrigatoriamente na primeira ou segunda infância; o comprometimento ou retardo do desenvolvimento de funções estreitamente ligadas à maturação biológica do sistema nervoso central e a evolução contínua sem remissões nem recaídas. Na maioria dos casos, as funções atingidas compreendem a linguagem, as habilidades espaço-visuais e a coordenação motora. Habitualmente o retardo ou a deficiência já estava presente mesmo antes de poder ser posta em evidência com certeza, diminuirá progressivamente com a idade; déficits mais leves podem, contudo, persistir na idade adulta.

Grupo 10 - Transtornos do comportamento e transtornos emocionais que aparecem habitualmente durante a infância ou a adolescência (F90-F98): Este grupo é constituído por transtornos hipercinéticos, distúrbios de conduta, transtornos mistos de conduta e das emoções, transtornos emocionais com início especificamente na infância, transtornos do funcionamento social com início especificamente durante a infância ou a adolescência, tiques e outros transtornos comportamentais e emocionais com início habitualmente durante a infância ou a adolescência.

Grupo 11 - Transtorno mental não especificado (F99): Grupo de transtornos mentais não classificados em outros agrupamentos.

\section{Resultados}

Dentre a população média de trabalhadores do estudo (2.181), foram identificados 2.931 afastamentos por motivos de saúde entre os anos de 2012 e 2016. Destes, 116 docentes apresentaram afastamentos por motivos de saúde mental, perfazendo 246 afastamentos. Assim, a prevalência de afastamentos do trabalho por TMCRT foi de $11 \%$ em relação à média de trabalhadores da educação do município e $8 \%$ em relação aos motivos de afastamento em geral. Quanto aos casos de afastamento, os resultados evidenciam que, dos 116 professores investigados, Perfil da amostra $94,8 \%$ são mulheres, $51,8 \%$ são casados, possuem filhos (75\%), possuem idade média de 44 anos e 9 meses (DP= 7,4) e tempo de trabalho de 16 anos e 4 meses $(\mathrm{DP}=13,3$ alto).

Entre 2012 e 2016 ocorreram 246 afastamentos tendo o de maior número ocorrido no ano de 2015 ( $\mathrm{n}=60,24,5 \%)$, conforme a ilustrado na Figura 1.

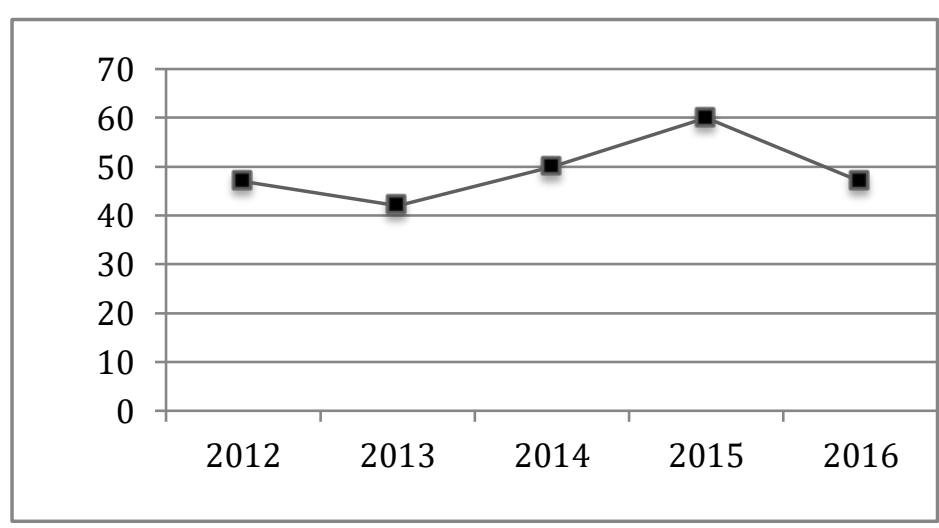

Figura 1. Taxas de afastamento do trabalho por Transtornos Mentais do Comportamento relacionados ao trabalho de professores, no período de 2012 a 2016. 
A figura 2 apresenta a série histórica dos motivos de afastamento indicando aumento do grupo F4 (Transtornos de Humor) e queda do grupo F5 (Transtornos neuróticos, transtornos relacionados com o estresse e transtornos somatoformes).

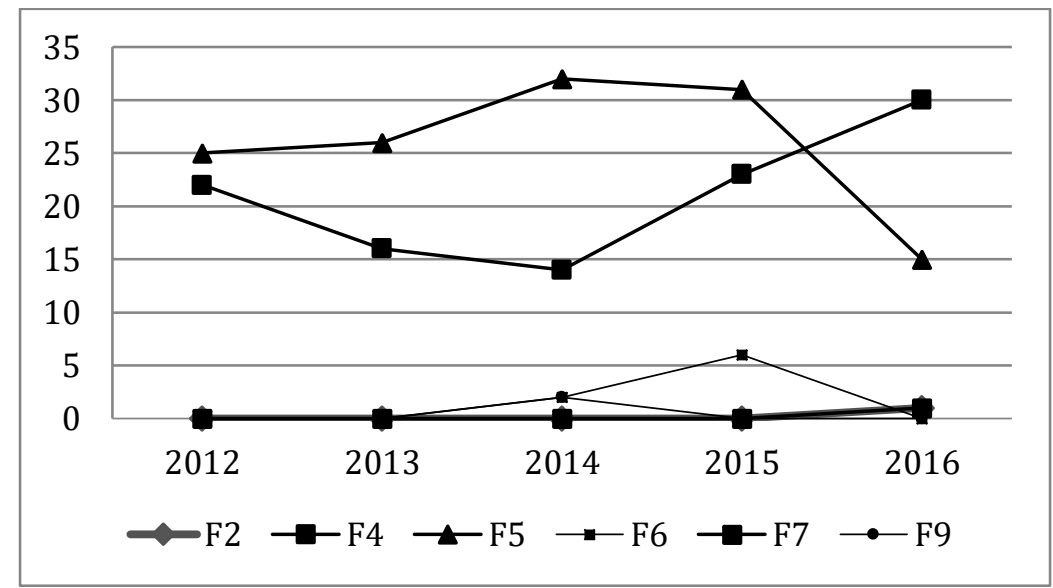

Figura 2. Taxas de afastamento do trabalho para tratamento de saúde de professores, segundo capítulos da CID 10, no período de 2012 a 2016.

Quanto aos grupos de Transtornos Mentais e do Comportamento, o Grupo 5, de Transtornos neuróticos, transtornos relacionados com o "stress" e transtornos somatoformes foi o que apresentou maior percentual de afastamentos $(52,4 \%)$, seguido pelos Transtornos de Humor $(42,7 \%)$ e os menores percentuais foram obtidos para o Grupo 9, de Transtornos do desenvolvimento psicológico e para o Grupo 7, de Transtornos da personalidade e do comportamento do adulto $(0,4 \%)$. Os grupos $1,3,8,10$ e 11 não apresentaram ocorrências (Tabela 1).

\section{Tabela 1}

Prevalência de afastamentos para tratamento de saúde por Transtornos Mentais e do Comportamento de professores, segundo capítulos da CID 10, no período de 2012 a 2016.

\begin{tabular}{lcc}
\hline \multicolumn{1}{c}{ CID - Transtornos mentais e comportamentais (F00-F99) } & f & \% \\
\hline F2. Transtornos mentais e comportamentais devidos ao uso de substância psicoativa (F10- & 1 & 0,4 \\
F19) & 105 & 42,7 \\
F4. Transtornos do humor (F30-F39) & 129 & 52,4 \\
F5. Transtornos neuróticos, transtornos relacionados com o “stress” e transtornos & 8 \\
somatoformes (F40-F48) & 3,3 \\
F6. Síndromes comportamentais associadas a disfunções fisiológicas e a fatores físicos & 1 \\
(F50-F59) & 2 & 0,4 \\
F7. Transtornos da personalidade e do comportamento do adulto (F60-F69) & 0,8 \\
F9. Transtornos do desenvolvimento psicológico (F80-F99) & $\mathbf{2 4 6}$ & $\mathbf{1 0 0}$ \\
\hline Total
\end{tabular}
sexo, idade, tempo de trabalho, dias de afastamento e diagnóstico médico (CID-10).

\section{Discussão}

O presente estudo buscou identificar a prevalência dos afastamentos do trabalho por TMCRT em professores entre os anos de 2012 e 2016. Quanto ao afastamento por TMCRT, verificou-se uma prevalência de $11 \%$ em relação à média de trabalhadores da educação do município e $8 \%$ em relação aos motivos de afastamento em geral. Os dados se aproximam aos encontrados em estudo realizado por Pizzio e Klein (2018) com servidores públicos federais do Tocantins $(7,10 \%)$ e dos identificados por Carvalho (2018) em professores da rede municipal de TeresinaPiauí $(9,2 \%)$. Os dados do presente estudo 
devem ser considerados como preocupantes, uma vez que avaliam diretamente TMCRT, os quais tendem a ser subnotificados em função de que ainda há desconhecimento médico sobre determinadas patologias laborais relacionadas aos transtornos mentais e do comportamento, bem como de seu nexo causal (Batista, Carlotto, Coutinho, \& Augusto, 2011; Carlotto, 2010).

Foram identificados dois grupos mais frequentes de afastamentos, o de Transtornos neuróticos, transtornos relacionados com o estresse e transtornos somatoformes $(52,4 \%)$ e o de Transtornos de humor $(42,7 \%)$. Os resultados estão alinhados a outros estudos sobre afastamentos por transtornos mentais em professores (Araújo \& Carvalho, 2009; Carvalho, 2018; Carlotto \& Câmara, 2015; Gasparini et al., 2006). O percentual obtido foi superior aos $41,1 \%$ encontrado em estudo realizado com professores por de Silva e Caveião (2016).

Quando os professores são investigados juntamente com outras categorias profissionais de servidores públicos são os que apresentam maior prevalência nestas modalidades de adoecimento (Silva, Tomé, Costa, \& Santana, 2012). Os resultados obtidos são preocupantes uma vez que trabalhadores que desenvolvem problemas de saúde mental geralmente apresentam comorbidades físicas (Zijlstra et al., 2006).

Embora os transtornos mentais possuam uma etiologia multicausal em que conjuntos de diversos fatores interagem de modo complexo (Jaques, 2007), pode-se considerar na contribuição dos estressores presentes no atual contexto educacional e organização do trabalho docente devido às categorias de afastamento dos professores investigados. Estudos têm revelado forte associação entre estressores ocupacionais e transtornos mentais em professores (Carlotto \& Câmara, 2015; Gasparini et al., 2006; Guglielmi \& Tatrow, 1998; Mayerl, Stolz, Waxenegger, Rásky, \& Freidl, 2016). O afastamento em professores possui um agravante relacionado às características do seu trabalho, com altas demandas cognitivas, pois segundo Zijlstra et al. (2006), quanto maiores as demandas cognitivas antes do absenteísmo, mais difícil o retorno, fazendo com que o trabalhador passe a considerar uma outra opção de trabalho.

Em termos da tendência de ocorrência ao longo dos anos, foi possível verificar um aumento de afastamentos entre os anos de 2013 e 2015, com uma diminuição nos afastamentos por TMCRT no ano de 2016. Em termos dos transtornos identificados como motivos de afastamento, na série histórica, houve um aumento dos Transtornos de Humor e queda dos Transtornos neuróticos, transtornos relacionados com o estresse e transtornos somatoformes.

Esses resultados devem ser interpretados com cautela, pois pode ter havido subnotificação real dos agravos de ordem mental em consequência da legislação vigente do Conselho Regional de Medicina - artigo 112/Resolução Conselho Federal de Medicina, no $1.658 / 2002$, pela qual, a colocação de CID, em atestado médico, só poderá ser feita mediante dever legal ou autorização expressa do paciente, sob pena do profissional violar o código de ética médica (Conselho Federal de Medicina, 2002). Pode-se supor que esta opção realizada pelo paciente está relacionada ao tipo de agravo, de natureza mental, devido ao estigma social ainda presente no contexto de trabalho.

Além disso, o modelo médico aborda a doença como o resultado da ação de agentes específicos, caracterizando-a como uma "doença geral" dissociada do contexto de trabalho (Laurell \& Noriega, 1989). Neste modelo, os afastamentos do trabalho são considerados como um problema médico, tendo em vista a maior visibilidade das demandas físicas que facilitam a sua definição, situação mais complexa no caso de adoecimentos mentais (Owens, 1997). Essas, exigem uma abordagem e análise interdisciplinar incluindo o médico do trabalho, o psicólogo do trabalho, o ergonomista, dentre outros segundo Lima (2005) e avaliadas a partir do contexto do contexto de trabalho onde 
ocorre o adoecimento (Landim, Bezerra, Alves, \& Marx, 2017).

Embora apresentem alta prevalência entre a população trabalhadora, os distúrbios psíquicos relacionados ao trabalho, não raras vezes, não são identificados na avaliação clínica. Este fato pode estar relacionado às próprias características dos distúrbios psíquicos, geralmente mascarados por sintomas físicos, e também pela complexidade inerente à tarefa de estabelecer claramente o nexo causal entre tais distúrbios e o trabalho (Amazarray, Câmara, \& Carlotto, 2014; Glina, Rocha, Batista, \& Mendonça, 2001).

Além da própria natureza do adoecimento mental, Cardoso e Araujo (2016) atribuem a subnotificação à falta de guias e protocolos para orientar os profissionais responsáveis pelo diagnóstico dos agravos para o estabelecimento do nexo causal entre o adoecimento e o trabalho. Esta situação, segundo os autores, deve ser superada, pois a adequada notificação dos agravos nos sistemas de informação de saúde é uma importante ferramenta para identificar o perfil de mortalidade dos trabalhadores e subsidiar ações efetivas para a promoção e prevenção em saúde do trabalhador. Cabe destacar também que, por vezes, o conhecimento do professor em relação a sua saúde mental, especificamente na abordagem dos riscos ocupacionais, ainda é incipiente, fazendo com que a busca por atendimento e diagnóstico ocorra somente em situações de maior gravidade.

\section{Conclusão}

A prevalência de TMCRT identificada indica um quadro grave de adoecimento dos professores que sugere a necessidade de monitoramento sistemático das taxas de absenteísmo por este tipo de agravos. Além disso, os resultados apontam a necessidade de investigação de fatores associados aos adoecimentos a fim de subsidiar medidas de promoção da saúde e prevenção de agravos à saúde dos professores.

O afastamento dos professores por problemas de saúde representa um custo econômico e social, na medida em que incide diretamente na qualidade da educação de uma comunidade. Miranda, Carvalho, Fernandes, Silva e Sabino (2009) pontuam que em âmbito individual, o afastamento do trabalho seja ele de caráter temporário e/ou definitivo, independente do motivo, ocasiona um impacto negativo na vida do trabalhador. Porém, na ocorrência de afastamentos devido a manifestações psicopatológicas dos transtornos mentais e comportamentais, não raras vezes, vem acompanhado de preconceito e a estigmatização. Soma-se a esta questão a presença limitações para a execução de atividades da vida diária, redução das potencialidades no contexto de trabalho e prejuízos da convivência familiar e social.

Diante desses aspectos, faz-se necessária a reflexão acerca do adoecimento mental relacionado ao trabalho enquanto um problema social. Embora o adoecimento se manifeste concretamente nos indivíduos, seus determinantes contextuais devem ser considerados, de maneira que se possa compreender e dimensionar o potencial adoecedor do trabalho. Esse tema precisa ser debatido na comunidade escolar (Carlotto \& Palazzo, 2006), entre trabalhadores em geral, sindicados, academia e a sociedade mais ampla, a fim de que seja possível identificar fatores de risco e promover saúde sem estigmatização (Silva, Bernardo, \& Souza, 2016).

Ações para a prevenção de adoecimentos mentais devem contemplar os fatores de estresse presentes no contexto de trabalho docente que, necessariamente, implicam em mudanças na organização do trabalho, processos de gestão e cultura institucional. Essas devem envolver gestores educacionais, equipe técnica, professores, alunos, pais/responsáveis e a sociedade em geral.

No entanto, a saúde docente deve ser contemplada em um âmbito mais amplo, em termos do projeto de educação de um país. Nesse sentido, o tema requer a implementação e o alinhamento de políticas públicas 
intersetoriais das áreas da saúde, do trabalho e da educação que contribuam para ambientes escolares mais saudáveis.

\section{Referências}

Affonso, S. L. (2016). Absenteísmo: um problema complexo nas organizações (Dissertação de mestrado). Curso de Mestrado em Administração, da Faculdade de Ciências Empresariais da Universidade - Fumec, Belo Horizonte, MG.

Aguiar, G. A. S., \& Oliveira, J. R. (2009). Absenteísmo. Suas principais causas e consequências em uma empresa do ramo de saúde. Revista de Ciências Gerenciais, 13(18), 95-113.

Amazarray, M. R., Câmara, S. G., \& Carlotto, M. S. (2014). Investigação em saúde mental e trabalho no âmbito da saúde pública no Brasil. In: A. R. C. Merlo, C. Bottega \& K. V. Perez. (Orgs.), Atenção à saúde mental do trabalhador: sofrimento e transtornos psíquicos relacionados ao trabalho. Porto Alegre: Evangraf.

Araújo, T. M., \& Carvalho, F. M. (2009). Condições de trabalho docente e saúde na Bahia: estudos epidemiológicos.

Educação e Sociedade, 30(107), 427-449. doi: 10.1590/S0101-73302009000200007

Alencar, M. C. B., \& Valença, J. B. M. (2016). Absence from work and functionality: the case of workers with lower back diseases. Caderno de Terapia Ocupacional da UFSCar, 24(4), 755-763. doi: 10.4322/0104-4931.ctoAO0732

Baasch, D., Trevisan, R. L., \& Cruz, R. M. (2017). Perfil epidemiológico dos servidores públicos catarinenses afastados do trabalho por transtornos mentais de 2010 a 2013. Ciência. \& Saúde Coletiva, 22(5). doi: $10.1590 / 1413-$ 81232017225.10562015

Batista, J. B. V., Carlotto, M. S., Coutinho, A. S., \& Augusto, L. G. D. S. (2010). Prevalência da Síndrome de Burnout e fatores sociodemográficos e laborais em professores de escolas municipais da cidade de João Pessoa, PB. Revista Brasileira de Epidemiologia, 13(3), 502-
512. doi: $10.1590 / \mathrm{s} 1415-$

790x2010000300013

Batista, J. B. V., Carlotto, M. S., Coutinho, A. S., \& Augusto, L. G. da S. (2011). Síndrome de Burnout: confronto entre o conhecimento médico e a realidade das fichas médicas. Psicologia em Estudo, 16(3), 429-435. doi: 10.1590/S141373722011000300010

Böckerman, P., \& Laukkanen, E. (2010). Predictors of sickness absence and presenteeism: does the pattern differ by a respondent's health? Journal of Occupational and Environmental Medicine, 52(3), 332-335. doi: 10.1097/jom.0b013e3181d2422f

Borrell, C., \& Artazcoz, L. (2008). Las desigualdades de género en salud: Retos para el futuro. Revista Espanhola de Salud Pública, 82(3), 241-249.

Recuperado de http://scielo.isciii.es/scielo.php?script=sci arttext\&pid=S113557272008000300001\&lng=es\&tlng=es.

Brasil. Ministério da Saúde do Brasil (2001). Doenças relacionadas ao trabalho: manual de procedimentos para os serviços de saúde. Brasília: MS.

Conselho Federal de Medicina (2002). Lei no 1.658/2002. Recuperado de http://portal.cfm.org.br/index.php?option= com_content\&id=2617

Cardoso, M. A. C. B., \& Araujo, T. M. (2016). Os Centros de Referências em Saúde do Trabalhador e as ações em saúde mental: um inquérito no Brasil. Revista. Brasileira de Saúde Ocupacional, 41, e7. doi: 10.1590/2317-6369000118115

Carlotto, M. S. (2010). O papel do psicólogo frente ao adoecimento e sofrimento ocupacional. Pessoas e Sintomas, 11, 4954. 
Carlotto, M. S. \& Câmara S. G. (2015). Prevalence and risk factors of common mental disorders among teachers. Journal of Work and Organizational Psychology 31, 201-206.

Carlotto, M. S., \& Palazzo, L. S. (2006). Síndrome de burnout e fatores associados: um estudo epidemiológico com professores. Caderno de Saúde Pública, 22(5), 1017-1026. doi: 10.1590/s0102-311x2006000500014

Carvalho, D. B. de (2018). Fatores associados ao absenteísmo por doença entre professores (Dissertação de Mestrado). Programa de Pós-Graduação em Saúde e Comunidade, Universidade Federal do Piauí.

Costa. S. S. (2017). Perfil Epidemiológico dos Transtornos Mentais Relacionados ao Trabalho na Região Nordeste do Brasil, no período de 2007 a 2016 (Trabalho de Conclusão de Especialização). Escola Bahiana de Medicina e Saúde Pública. Salvador, Bahia.

Costa, F. R. C. P., \& Rocha, R. (2013). Fatores estressores no contexto de trabalho docente. Revista Ciências Humanas -UNITAU 6(1), 18-43.

Estorce, T. P., \& Kurcgan, T. P. (2011). Licença médica e gerenciamento de pessoal de enfermagem. Revista da Escola de Enfermagem da USP, 45(5), 11991205. doi: $10.1590 / \mathrm{S} 0080-$ $\underline{62342011000500024}$

Falavigna, A., \& Carlotto, M. S. (2013). Tendência temporal de afastamento do trabalho por transtornos mentais e comportamentais em enfermeiros (19982008). Revista Psicologia Organizações e Trabalho, 13(3), 363-371.

Gasparini, S. M., Barreto, S. M. \& Assunção, A. A. (2006). Prevalência de transtornos mentais comuns em professores da rede municipal de Belo Horizonte, Minas Gerais, Brasil. Caderno de Saúde Pública, 22(12), 2679-2691. doi: 10.1590/s0102$\underline{311 \times 2006001200017}$
Glina, D. M. R., Rocha, L. E., Batista, M. L., \& Mendonça, M. G. V. (2001). Saúde mental e trabalho: uma reflexão sobre o nexo com o trabalho e o diagnóstico, com base na prática. Cadernos de Saúde Pública, 17(3), 607-616. doi: 10.1590/S0102-311X2001000300015

Guglielmi, R. S., \& Tatrow, K. (1998). Occupational stress, burnout, and health in teachers: a methodological and theoretical analysis. Review of Educational Research, 68(1), 61-99. doi: $\underline{10.3102 / 00346543068001061}$

Jaques, T. (2007). Issue management and crisis management: An integrated, nonlinear, relational construct. Public Relations Review, 33(2), 147-157. doi: 10.1016/j.pubrev.2007.02.001

Khan, M. S. (2014). Teacher burnout - Causes and prevention. Vetri Education, IX(2), 15-22.

Khan, A., Amanat, A., Aqeel, M., Sulehri, N. A., Sana, E., Amin, H., \& Amanat, A. (2017). The mediating role of job stress between social support and development of stress, anxiety and depression in educators and health professionals. Foundation University Journal of Psychology, 1(3), 48-61.

Landim, J. M. M., Bezerra, M. M. M., Alves, M. N. T., \& Marx, M. (2017). Saúde Mental do Trabalhador no Brasil: Questões emergentes. Id on line Revista Multidisciplinar e Psicologia, 30(33), 186-197.

Laurell, A. C., \& Noriega, M. (1989). Processo de produção e saúde: trabalho e desgaste operário. São Paulo: Hucitec.

Leão, A. L. M., Barbosa-Branco, A., Rassi Neto, E., Ribeiro, C. A. N, \& Turchi, M. D. (2015). Absenteísmo-doença no serviço público municipal de Goiânia. Revista Brasileira de Epidemiologia, 18(1), 262-277.

Lima, M. E. A. (2005). Transtornos mentais e trabalho: o problema do nexo casual. Revista de Administração da FEADMinas, 2(1), 73-80. 
Maia, P. A. (2014). Trabalho docente e licenças médicas de professores afastados. Revista Montagem, 16(16), 145-156.

McDaid, D. (2008). Mental health in workplace settings. Luxembourg: European Commission.

Mayerl, H., Stolz, E., Waxenegger, A., Rásky, É., \& Freidl, W. (2016). The role of personal and job resources in the relationship between psychosocial job demands, mental strain, and health problems. Frontiers in Psychology, 7, 1214. doi: 10.3389/fpsyg.2016.01214 Melo, W. F., Rego, S. M. O., Saldanha, H. G. A. C., Flor, M. F. P. C. O., \& Maracaja, P. B. (2015). Síndrome de Burnout em professores. Revista Brasileira de Educação e Saúde, 5(4), 01-06.

Miranda, F. A. N., Carvalho, G. R. P., Fernandes, R. L., Silva, M. B., \& Sabino, M. G. G. (2009). Saúde mental, trabalho e aposentadoria: focalizando a alienação mental. Revista Brasileira de Enfermagem, 62(5), 711-716. doi: 10.1590/S0034-71672009000500010

Morais, L. A. A., Souza, K. R. de, \& Santos, G. B. (2018). Intensificação e precarização social do trabalho de professores de escola pública: um estudo exploratório na região da baixada fluminense (RJ). Revista Trabalho Necessário, 16(29), 218-236.

Munro, L. (2007). Absenteeism and presenteeism: possible causes and solutions. The South African Radiographer, 45(1), 21-23.

Organização Mundial de Saúde [OMS]. (1993). Classificação de transtornos mentais e de comportamento: CID-10. Porto Alegre: Artes Médicas.

Owens, P. (1997). Mental health: understanding work disability issue. Health Insurance Underwriter, 1-4.

Pizzio, A., \& Klein, K. B. (2018). Perfil epidemiológico dos servidores públicos federais no Tocantins afastados por motivo de saúde. Revista Brasileira de Gestão e Desenvolvimento Regional, 14(2), 588-604.
Sales, L. O., \& Freitas, M. C. S. (2018). A experiência com o adoecimento na docência: um estudo com professoras do município de São Miguel das Matas, Bahia, Brasil. Sisyphus Journal of Education, 6(02),65-81.

Santi, D. B., Barbieri, A. R., \& Cheade, M. F. M. (2018). Absenteísmo-doença no serviço público brasileiro: uma revisão integrativa da literatura. Revista Brasileira de Medicina do Trabalho, 16(1),71-81. doi: 10.5327/Z1679443520180084

Santos, J. K. L., \& Wanzinack, C. (2017). Saúde docente: um estudo de caso nas escolas municipais de ensino fundamental do município de Matinhos - Paraná Brasil. Revista Eletrónica de Investigación y Docencia, 17, 115-128. doi: 10.17561/reid.v0i17.3187

Schreuder, J. A., Roelen, C. A., Koopmans, P. C., Moen, B. E., \& Groothoff, J. W. (2010). Effort-reward imbalance is associated with the frequency of sickness absence among female hospital nurses: a cross-sectional study. International Journal of Nursing Studies, 47(5), 569576. doi: 10.1016/j.ijinurse.2009.10.002

Seligmann-Silva, S. E. (2009). Saúde mental no trabalho contemporâneo. In congresso Internacional de Stress da ISMA-BR (vol. 9).

Seligmann-Silva, E., Bernardo, M. H., Maeno, M., \& Kato, M. (2010). Saúde do Trabalhador no início do século XXI (Editorial). Revista Brasileira de Saúde Ocupacional, 35(122), 185-186. doi: 10.1590/s0303-76572010000200001

Silva, M. P., Bernardo, M. H., \& Souza, H. A. (2016). Relação entre saúde mental e trabalho: a concepção de sindicalistas e possíveis formas de enfrentamento.

Revista Brasileira de Saúde Ocupacional, 41, 23. doi: $\underline{10.1590 / 2317-}$ 6369000003416

Silva, E. B. F., Tomé, L. A. O., Costa, T. J. G., \& Santana, M. C. C. P. (2012).

Transtornos mentais e comportamentais: perfil dos afastamentos de servidores públicos estaduais em Alagoas, 2009. 
Epidemiologia e Serviços de Saúde, 21(3), 505-514. doi: $10.5123 / \mathrm{s} 1679$ -

49742012000300016

Silva, M. M., \& Caveião, C. (2016). Análise dos afastamentos de saúde dos trabalhadores de ensino de Divinópolis MG. Revista Saúde e Desenvolvimento, 10(5), 138-156.

Silva Junior, J. S., \& Fischer, F. M. (2014). Adoecimento mental incapacitante: benefícios previdenciários no Brasil entre 2008-2011. Revista de Saúde Pública, 48(1), 186-190. doi: 10.1590/S0034$\underline{8910.2014048004802}$

Soldatelli, R. (2011). O processo de adoecimento dos professores do ensino fundamental de Florianópolis e suas possibilidades de resistência a esse processo (Dissertação de Mestrado). Florianópolis, SC.

Sprenger, J. (2011). Stress and coping behaviors among primary school teachers. Master in Arts. Faculty of the Department of Health Education and Promotion. East Carolina University. EUA. Recuperado de http://thescholarship.ecu.edu/bitstream/ha ndle/10342/3548/Sprenger_ecu_0600M_1 0405.pdf?seq

Stansfeld, S. A., North, F. M., White, I., \& Marmot, M. G. (1995). Work characteristics and psychiatric disorder in civil servants in London. Journal of Epidemiology \& Community

Health, 49(1), 48-53. doi: 10.1136/jech.49.1.48

Ybema, J. F., Smulders, P. G., \& Bongers, P. M. (2010). Antecedents and consequences of employee absenteeism: A longitudinal perspective on the role of job satisfaction and burnout. European Journal of Work and Organizational Psychology, 19(1), 102-124. doi: 10.1080/13594320902793691

Wynne, R. et al. (2014). Employment, social affairs \& inclusion. Promoting mental health in the workplace: guidance to implementing a comprehensive approach. European Commission. Recuperado de ec.europa.eu/social/BlobServlet?docId=13 $\underline{879 \& \text { langId }=\text { en }}$

Zijlstra, F. et al. (2006). Stress Impact project. Stress Impact is the acronym for the EU funded project entitled: The impact of changing social structures on stress and quality of life: Individual and social perspectives. Recuperado de https://www.surrey.ac.uk/psychology/proj ects/stressimpact/files/Stress\%20Impact\%20Integrat ed\%20Report.pdf

\section{Dados sobre os autores:}

- Mary Sandra Carlotto: Graduação em Psicologia pela Universidade do Vale do Rio dos Sinos (1983), especialização em Gestão de Recursos Humanos pela Universidade Cândido MendesRJ (2000), mestrado em Saúde Coletiva pela Universidade Luterana do Brasil (2002) e doutorado em Psicologia Social pela Universidade de Santiago de Compostela (2005). Professora da Universidade do Vale do Rio dos Sinos-UNISINOS. Coordenadora do Grupo de Pesquisa em Psicologia da Saúde Ocupacional (GPPSO).

- Sheila Gonçalves Câmara: Psicóloga; Mestre e Doutora em Psicologia (PUCRS). Docente do PPG Psicologia e Saúde da Universidade Federal de Ciências da Saúde de Porto Alegre (UFCSPA).

- Jaqueline Vidal Batista: Psicóloga, Especialista em Teoria Psicanalítica (UFPB); Mestre em Psicologia Social (UFPB); Doutora em Saúde Pública (FIOCRUZ); Professora do PPG em Enfermagem da Universidade Federal da Paraíba (UFPB).

- Gabriela Azeredo Schneider: Bolsista de Iniciação Científica (UNIBIC/UNISINOS). 